\title{
A new self-scaling VM-algorithm with an adaptive line search
}

\author{
Abbas. Al-Baiati Salah G. Shareef \\ Hamsa Th. Chilmerane \\ Department of Mathematics \\ College of Computers Sciences \& Mathematics \\ Mosul University
}

Received

ro / 0 T / 2008
Accepted

16 / 02 / 2009

في هذا البحث م اقتراح خوارزمية جدية للمتري المتغير ذلتي القيلس في الامثلية غير الخطية وغير المقية مع لستخدله غط بهث ملأم وبعد لمد تخدلم ص فه الق ارب المحل ي لمصفوفة BFGS للحصول على صفه القارب الشلمل في المصفوفة ألمستخلمة. لق سـ ت م مقارنه هذه الخوارزمية مع خوارزمية BFGS القيلسية وكالت النتائج مشجعة جداً.

\begin{abstract}
In this study, a new self-scaling VM algorithm for solving largescale nonlinear unconstrained optimization problems is proposed. The new algorithm is a kind of line search procedure. The local convergence theory for the BFGS matrix implementation of this variable metric is applied to achieve a globally convergent property.Numerical experiments have been done with the standard BFGS algorithm with very promising results.
\end{abstract}

Keywords: Self-scaling Variable Metric, Line search procedure, Largescale optimization, Global convergence algorithm. 


\section{Introduction}

consider line search descent method for solving the unconstrained optimization problem

$$
\min _{x \in R^{n}} f(x)
$$

where $f$ is twice continuously differentiable. Each method generates a sequence of points $\left\{x_{k}\right\}$ for $k=1,2, \ldots \ldots \ldots$ until termination. The initial point $x_{i}$ is given. If $g_{k}=\nabla f\left(x_{k}\right)=0$, for some $k$, then the method terminates with $x_{k}=x^{*}$. Otherwise, a search direction $d_{k}$ is defined for which

$$
g_{k}^{T} d_{k}<0
$$

(the descent property). Then a new iterate is defined by the line search

$$
x_{k+1}=x_{k}+\lambda_{k} d_{k}
$$

where $\lambda_{k}$ is a step length chosen to minimize $f$ along $\mathrm{d}_{k}$ for which

$$
g_{k+1}^{T} d_{k}=0
$$

In this case the line search is exact (see [1]). The search direction of Newton's method is defined by

$$
d_{k}=-G_{k}^{-1} g_{k}
$$

where $G_{k}=\nabla^{2} f\left(x_{k}\right)$ is the Hessian matrix which, for $x_{k}$ sufficiently close to $x^{*}$, is usually positive definite. The methods that we study are all iterative methods, since in the quadratic case we cannot expect to be able to carry out enough iteration to obtain an exact solution, even if the theory allows this possibility, due to the size of $n$ or the build up of round off error. For the quadratic case the conjugate gradient (CG) method itself (see [3]), or some preconditioned conjugate gradient (PCG) method (see[4]), is usually the method of choice, although there are other variants such as the minimum residual (MR) algorithm that are also applicable to the case that $\mathrm{G}$ is symmetric and indefinite. We consider the case when a preconditioned version of a nonlinear conjugate gradient method is used to minimize $f$; the matrix $\bar{G}$ will denote the precondition matrix. At the first iteration, the search direction is defined by

$$
\begin{array}{r}
d_{1}=-\bar{G}^{-1} g_{1} \quad \ldots \ldots \ldots \ldots \\
\quad \quad \text { for } k>0, \\
d_{k}=-\bar{G}_{k}^{-1} g_{k}+\beta_{k-1} d_{k-1}
\end{array}
$$

where

and where

$$
\beta_{k+1}=\frac{y_{k-1}^{T} \bar{G}_{k}^{-1} g_{k}}{y_{k-1}^{T} d_{k-1}}
$$




$$
y_{k-1}=g_{k}-g_{k-1}
$$

(This method is referred to as preconditioned conjugate gradient (PCG), for more details (see[1])). Resetting corresponds to defining $d_{k}$ for $k>1$ as

$$
d_{k}=-\bar{G}_{k}^{-1} g_{k}
$$

which in effect starts a new sequence of iteration. For the VM-methods assume that at the $k$ th iteration at approximation point $x_{\mathrm{k}}$ and $n \times n$ matrix $H_{k}$ are available. Then the methods proceed by generating a sequence of approximation points via the equation:

and

$$
x_{k+1}=x_{k}+\lambda_{k} d_{k}
$$

$$
d_{k+1}=-H_{k} g_{k}
$$

where $H_{k}$ is an approximation of $G^{-1}$ which is corrected or updated form iteration to iteration, in general $H_{k}$ is symmetric and positive definite. There are different choice of $H_{k}$ (see[5]) we list here some most popular forms (see[6])

$$
H_{k+1}^{S R}=H_{k}+\frac{\left(v_{k}-H_{k} y_{k}\right)\left(v_{k}-H_{k} y_{k}\right)^{T}}{\left(v_{k}-H_{k} y_{k}\right)^{T} y_{k}}
$$

is called rank one correction formula, where $v_{k}=x_{k+1}-x_{k}$ and $y_{k}$ is defined as in equation(9)

$$
H_{k+1}^{B F G S}=H_{k}+\left[1+\frac{y_{k}^{T} H_{k} y_{k}}{v_{k}^{T} y_{k}}\right] \frac{v_{k} v_{k}^{T}}{v_{k} y_{k}^{T}}-\left[\frac{v_{k} y_{k}^{T} H_{k}+H_{k} y_{k} v_{k}^{T}}{v_{k} y_{k}^{T}}\right]
$$

which are satisfies the Quasi-Newton condition defined by

$$
H_{k+1} y_{k}=v_{k}
$$

and maintains the positive definite matrices if $H_{0}$ is positive.

\section{Self-scaling VM methods}

To improve the performance of VM updates, (see [6]) we are trying to choose $H_{k+1}$ to satisfy the following modified QN equation

$$
H_{k+1} y_{k}=\rho_{k} v_{k}
$$

where $\rho_{k}>0$ is a scaling parameter . The BFGS may be written as:

$$
H_{k+1}=H_{k}-\frac{H_{k} y_{k} v_{k}^{T}+v_{k} y_{k}^{T} H_{k}}{v_{k}^{T} y_{k}}+\left[\frac{1}{T_{k}}+\frac{y_{k}^{T} H_{k} y_{k}}{v_{k}^{T} y_{k}}\right] \frac{v_{k} v_{k}^{T}}{v_{k}^{T} y_{k}}
$$

where

$$
T_{k}=\frac{1}{\lambda_{k}}=\frac{6}{v_{k}^{T} y_{k}}\left(f\left(x_{k}\right)-f\left(x_{k+1}\right)+v_{k}^{T} g_{k+1}\right)-2
$$


in fact the self-scaling update proposed by (Oren [8]) has some good characteristics, with a self -scaling parameter $\gamma_{k}$, this class of updates can be written as

$$
\begin{aligned}
H_{k+1}= & {\left[H_{k}-\frac{H_{k} y_{k} y_{k}^{T} H_{k}}{v_{k}^{T} H_{k} y_{k}}+\phi_{k}\left(y_{k} H_{k} y_{k}\right) v_{k}^{-} v_{k}^{-T}\right] \gamma_{k}+\frac{v_{k} v_{k}^{T}}{v_{k}^{T} y_{k}} } \\
& \text { with } \bar{v}=\frac{v_{k}}{v_{k}^{T} y_{k}}-\frac{H_{k} y_{k}}{y_{k}^{T} H_{k} y_{k}}
\end{aligned}
$$

(Oren and Luenberger [9]) suggest to use the self- adaptable values for the parameter $\gamma_{k}$

$$
\gamma_{k}=t \frac{g_{k}^{T} v_{k}}{g_{k}^{T} H_{k} y_{k}}+(1-t) \frac{v_{k}^{T} y_{k}}{y_{k}^{T} H_{k} y_{k}}
$$

and usually the value $t=0$ is recommended for the updates in the convex class, i.e

$$
y_{k}=\frac{v_{k}^{T} y_{k}}{y_{k}^{T} H_{k} y_{k}} \text { and } \sigma_{k}=\frac{1}{\gamma_{k}}=\frac{y_{k}^{T} H_{k} y_{k}}{v_{k}^{T} y_{k}} \text { (AL-Bayti 1991) }
$$

\section{Armijo line search procedure}

Armijo provided in [10] a modification of the steepest descent method which automatically adapts step size $\lambda$ of the iterative scheme

$$
x_{k+1}=x_{k}-\lambda_{k} \nabla f\left(x_{k}\right), k=1,2,3,
$$

his method and the corresponding convergence result are as follows:

\section{Theorem (Armijo[10])}

suppose that the objective function $f: R^{n} \rightarrow R$ is continues on $\mathrm{R}^{\mathrm{n}}$ and bounded below on $R^{n}$. Assume that for a given $x_{0} \in R^{n}$, the function $f$ is continuously differentiable on the bounded level set $\bar{\alpha}\left(x_{0}\right)=\left\{x: f(x) \leq f\left(x_{0}\right\}\right.$ and that there exists a unique point $x^{*} \in R^{n}$ which minimize $f$. Suppose further that the equation $\nabla f(x)=0$ is satisfied for $x_{o} \in \bar{\alpha}\left(x_{o}\right)$ if and only if $x=x^{*}$ and that $\nabla f$ is Lipschitz continues on $\bar{\alpha}\left(x_{0}\right)$, i.e, there exists a Lipschitz constant $k \neq 0$, such that

$$
\|\nabla f(x)-\nabla f(y)\| \leq k\|x-y\|,
$$

for every pair $x, y \in s\left(x_{0}\right)$. Let $\lambda_{0}$ be an arbitrary assigned positive number,and consider the sequence $\lambda^{m}=\lambda^{m} / 2^{m-1}, m=1,2, \ldots \ldots \ldots$. Then for the sequence of points $\left\{x_{k}\right\}_{k=0}^{\infty}$ 
defined by

$$
x_{k+1}=x_{k}-\lambda_{k}^{T} \nabla f(x), \quad \mathrm{k}=0,1,
$$

where $m$ is the smallest positive integer for which

$$
f\left(x_{k}-\lambda_{k}^{m} \nabla f\left(x_{k}\right)\right)-f\left(x_{k}\right) \leq-\frac{1}{2} \lambda_{k}^{m}\left\|\nabla f\left(x_{k}\right)\right\|^{2}
$$

it holds that $\lim _{k \rightarrow \infty} x=x^{*}$

Next, a high-level description of the Armijo's algorithm, in which the corresponding parameters indicate $x_{0}$ initial point, $\lambda_{0}$ an arbitrary large initial step size, NIT (number of iterations) the maximum number of iterations required and $\in$ the predetermined desired accuracy.

\section{Algorithm1}

1- Input $\left\{f: x_{0}: \lambda_{0} ; \mathrm{NIT}, \varepsilon\right\}$

2- Set $k=1$

3- If $k<$ NIT, replace $k$ by $k+1$, set $\lambda=\lambda_{0}, m=1$ and go to next step: otherwise, go to $\operatorname{step}(8)$

4- If $f\left(x_{k}-\lambda \nabla f\left(x_{k}\right)\right)-f\left(x_{k}\right) \leq-\frac{1}{2} \lambda\left\|\nabla f\left(x_{k}\right)\right\|^{2}$, go to step (6); otherwise, set $m=m+1$ and go to the next step.

5- Set $\lambda=\lambda_{0} / 2^{m-1} *$ and return to step (4).

6- Set $x_{k+1}=x_{k}-\lambda \nabla f\left(x_{k}\right)$

7- If $\left\|\nabla f\left(x_{k}\right)\right\| \leq \varepsilon$ go to step(8); otherwise go to step (3).

8- Output $\left\{x_{k} ; f\left(x_{k}\right) ; \nabla f\left(x_{k}\right)\right\}$

\section{A new self-scaling VM- update}

In this section a new self-scaling VM algorithm is proposed, with modified Armijo line search, and so we develop a Preconditioned CGalgorithm. This new approach finds the minimum of an $n$ dimensional non quadratic function. Now let the $\mathrm{g}_{\mathrm{n}}$ is denoted as the gradient of the function $f\left(x_{k}\right)$. For simplicity $f_{k}$ and $G_{k}$ denote $f\left(x_{k}\right)$ and $\nabla^{2} f\left(x_{k}\right)$, respectively.

Using the Taylor expansion to the third-order terms $f$ and $g^{T}$ can be written as respective

$$
f_{k}=f_{k+1}-g_{k+1}^{T} v+\frac{1}{2 !} v^{T} G_{k+1} v_{k}-\frac{1}{3 !} v_{k}^{T}\left(T_{k+1} v_{k}\right) v_{k}+o\left\|v_{k}\right\|^{4}
$$




$$
g_{k}^{T} v_{k}=g_{k+1}^{T} v_{k}-v_{k}^{T} G_{k+1} v_{k}+\frac{1}{2 !} v_{k}^{T}\left(T_{k+1} v_{k}\right) v_{k}+o\left(\left\|v_{k}\right\|^{4}\right)
$$

where $T_{k+1} \in R^{n \times n \times n}$ is the tensor of $f$ at $x_{k+1}$. We obtain the following relation by canceling the terms which induce the tensor in above expressions

$$
v_{k}^{T} G_{k+1} v_{k}=v_{k}^{T} y_{k}+6\left(f_{k}-f_{k+1}\right)+3\left(g_{k}+g_{k+1}\right)^{T} v_{k}+o\left\|v_{k}\right\|^{4}
$$

Then using a new approximation $B_{k+1}$, we have, after putting $o\left\|v_{k}\right\|^{4}$ (small value),

where

$$
v_{k}^{T} B_{k+1} v_{k}=v_{k}^{T} y_{k}+\theta_{k}
$$

$$
\theta_{k}=6\left(f_{k}-f_{k+1}\right)+3\left(g_{k}+g_{k+1}\right)^{T} v_{k}
$$

equation (23) givens a class of modified secant condition in the form

$$
y_{k}^{-}=y_{k}+\frac{\theta_{k}}{v_{k}^{T} u_{k}} u_{k}
$$

where $u_{k} \in R^{n}$ is any vector such that $v_{k}^{T} u_{k} \neq 0$.

Now from (17),replacing (24) instead of $y_{k}$ then we get

$$
H_{k+1}=H_{k}-\frac{H_{k} \bar{y}_{k} v_{k}^{T}+v_{k} \bar{y}_{k}^{T} H_{k}}{v_{k}^{T} \bar{y}_{k}}+\left(\frac{1}{T}+\frac{\bar{y}_{k} H_{k} \bar{y}_{k}}{v_{k}^{T} \bar{y}_{k}}\right) \frac{v_{k} v_{k}^{T}}{v_{k}^{T} y_{k}}
$$

And by Armijo line search with $\lambda_{\mathrm{k}}$ is the largest number in $\left\{s, s / 2, s / 2^{2}, \ldots \ldots \ldots \ldots . . ..\right\}$ such that

$f_{k}-f\left(x_{k}+\lambda d_{k}\right) \geq-\eta \lambda g_{k}^{T} d_{k}$ Where $\eta \in(0,1)$, and $s \neq 0$

Note 1 :In fact, we can use the generalized Armijo's line search $\lambda_{\mathrm{k}}$ is the largest number in $\left\{s, s \beta, s \beta^{2}, s \beta^{3}, \ldots \ldots\right\}$ such that

$$
f_{k}-f\left(x_{k}+\lambda_{k} d_{k}\right) \geq-\eta_{1} \lambda g_{k}^{T} d_{k} \text { where } \eta_{1} \in(0,1), \beta \in(0,1) \text {, and } s \neq 0
$$

Note 2: we should choose a suitable step size at each iteration so that the Armijo, type line search on $\bar{\theta}$ is then carried out until the acceptance condition

$$
f\left(x_{k}+d_{k}\right) \leq \max _{\max (k-m) \leq j \leq k} f(j)-\eta g_{k}^{T} d
$$

is met, where $d=-\bar{\theta} g_{k}$ is the displace element along the steepest direction where

$$
\eta=10, \eta \in(0,1), \bar{\theta}=1, \frac{1}{10}, \frac{1}{100}
$$




\section{The Global convergence property of the new proposed algorithm}

Let $\mathrm{s}$ be a positive parameter and let $x_{s}=x_{k}-s d_{k}$ where $d_{k}=-H_{k} g_{k}$

Armijo's rule for determining $\lambda$ is the following evaluate $\left\|g\left(x_{s}\right)\right\|$ at $s=1, \frac{1}{2}, \frac{1}{4}$

Stopping when

$$
\left\|g\left(x_{s}\right)\right\| \leq\left(1-\frac{s}{2}\right)\left\|g\left(x_{k}\right)\right\|
$$

if $\lambda$ denotes the first $s$ which satisfies (27), then $x_{k+1}$ is given by

$$
x_{k+1}=x_{k}-\lambda H_{k} g_{k}
$$

usually, the Euclidean norm is used in (27) and the sum of the squares of g's components are reduced at each iteration.

The problem (1), at the minimum when $g(x)=0$, the partial derivative respect to each of the unknown vanishes:

$$
\sum_{i=1}^{n} \frac{\partial f_{i}}{\partial x_{j}}(x) f_{i}(x)=0
$$

For $j=1,2, \ldots \ldots \ldots \ldots \ldots \ldots, n$

Using matrix-vector notation (29) is written as $j(x)^{T} g(x)=0$. If $\mathrm{j}(\mathrm{x})$ is invertible the equation $j(x)^{T} g(x)=0$ implies that any minimizer for (1) satisfies $g(x)=0$.

\section{Outline of new self-scaling VM algorithm with Armijo line search}

Step (1): set the initial point $x_{0}$, the scalar $\varepsilon$ and $H_{0}=I, \beta=1, k_{1}=1$

Step (2): for $\mathrm{k}=1$ set $d_{1}=-H_{1} g_{1}$ where $g_{1}=\nabla f\left(x_{1}\right)$

Step (3): compute $g_{k}^{T} g_{k}$

Step(4): if $(k \neq 1)$ go to step (5)

$$
\begin{aligned}
& \mathrm{B}=\lambda \\
& \text { go to step (6) }
\end{aligned}
$$

Step(5): if $(\mathrm{k}=2)$

$$
\begin{aligned}
& \bar{\beta}=0.2 \\
& \lambda=\bar{\beta}
\end{aligned}
$$

goto step (6) 
step(6): compute $x_{k+1}=x_{k}+\lambda_{k} d_{k}$ where $\lambda_{k}$ is obtained from Armijo line search procedure.

Step(7): if $f_{k+1}-f_{k} \leq 0.1 \lambda\left(g_{k+1} d_{k}-0.5 \lambda g_{k} d_{k}\right)$ go to step (8).

Otherwise set

$$
k=k+1 \text { and go to step (4) }
$$

step(8): set $\quad v_{k}=x_{k+1}-x_{k}$ and $y_{k}=g_{k+1}-g_{k}$

step (9): compute $\theta_{k}$ where

$$
\begin{aligned}
& \theta_{k}=6\left(f_{k}-f_{k+1}\right)+3\left(g_{k}-g_{k+1}\right)^{T} v_{k} \\
& \bar{y}=y_{k}+\frac{\theta_{k}}{v_{k}^{T} y_{k}} y_{k}
\end{aligned}
$$

step (10): compute $T_{k}$ from (20)

$$
\bar{\tau}_{k}=\frac{\bar{y}_{k}^{T} H_{k} \bar{y}}{v_{k}^{T} \bar{y}_{k}}
$$

step(11): if $\left(\bar{\tau}_{k} \neq 0.5 \cdot O R \cdot \bar{\tau}_{k} \neq \frac{1}{\tau_{k}}\right)$

then

$$
\begin{aligned}
& \phi=\frac{1}{\tau_{k}} \\
& \bar{\tau}_{k}=1
\end{aligned}
$$

step(12): compute $H_{k+1}$

$$
H_{k+1}=H_{k}-\frac{H_{k} \bar{y}_{k} y_{k}^{T} H_{k}}{\bar{y}_{k}^{T} H_{k} \bar{y}_{k}}+\phi_{k}\left(y_{k} H_{k} y_{k}\right) \bar{v}_{k} \bar{\nu}_{k}^{T}+\bar{\tau}_{k} \frac{v_{k} v_{k}^{T}}{v_{k}^{T} y_{k}}
$$

where $\bar{v}_{k}=\frac{v_{k}}{v_{k}^{T} \bar{y}_{k}}-\frac{H_{k} \bar{y}_{k}}{\bar{y}_{k}^{T} H_{k} \bar{y}_{k}}$

step (13): $d_{k+1}=-H_{k+1} g_{k+1}+\frac{\bar{y}_{k} H_{k+1} g_{k+1}}{d_{k}^{T} \bar{y}_{k}} d_{k}$

step(14): if $k=N$ or $g_{k}^{T} g_{k} \leq g_{k+1}^{T} g_{k+1}$ then go to step (2)

else go to step (3) 


\section{Numerical Results}

The following table, gives the comparison between the results of the new self-scaling VM algorithm with Armijo line search and standard BFGS algorithm. In the all cases the stopping criterion is $\left\|g_{k+1}\right\| \leq 1 \times 10^{5}$. Also we run the program on a set of test functions with five versions of variables, that is $n=4,20,100,500$ and 1000 .

The results are given in the table is specifically quoting the number of function evaluations NOF and the number of iterations NOI. Experimental results confirm that the new algorithm is superior to the standard BFGS algorithm .From the Table, taking the standard BFGS algorithm as $100 \%$ NOI, and NOF respectively, there are about $47 \%$ NOI and $55 \%$ NOF improvement overall selected group of test functions.

Comparative performance of the two algorithms (classical BFGS with cubic line search and new self-scaling VM with Armijo line search)

\begin{tabular}{|l|l|l|}
\hline \multicolumn{1}{|c|}{ Test function } & Classical BFGS & New self-scaling VM \\
\cline { 2 - 3 } & NOF (NOI) & NOF (NOI) \\
\hline Powell(4) & $22(71)$ & $32(44)$ \\
\hline Powell(20) & $40(117)$ & $39(69)$ \\
\hline Powell(100) & $71(197)$ & $64(155)$ \\
\hline Powell(500) & $50(148)$ & $49(114)$ \\
\hline Powell(1000) & $62(155)$ & $137(329)$ \\
\hline Wood(4) & $55(145)$ & $24(46)$ \\
\hline Wood(20) & $130(358)$ & $49(114)$ \\
\hline Wood(100) & $262(746)$ & $105(278)$ \\
\hline Wood(500) & 583() & $148(371)$ \\
\hline Wood(1000) & $692(1792)$ & $234(422)$ \\
\hline Cubie(4) & $19(59)$ & $11(25)$ \\
\hline Cubie(20) & $27(66)$ & $24(73)$ \\
\hline Cubie(100) & $70(167)$ & $30(94)$ \\
\hline Cubie(500) & $53(124)$ & $31(100)$ \\
\hline Cubie(1000) & $44(112)$ & $28(88)$ \\
\hline Rosen $(4)$ & $35(94)$ & $18(36)$ \\
\hline Rosen (20) & $70(180)$ & $34(89)$ \\
\hline Rosen (100) & $139(353)$ & $49(161)$ \\
\hline Rosen (500) & $88(192)$ & $66(214)$ \\
\hline Rosen (1000) & $92(252)$ & $64(208)$ \\
\hline Dixon (4) & $11(21)$ & $11(21)$ \\
\hline Dixon (20) & $11(21)$ & $30(56)$ \\
\hline Dixon (100) & $11(21)$ & $37(75)$ \\
\hline Dixon (500) & $11(21)$ & $38(77)$ \\
\hline Dixon (1000) & $11(21)$ & $39(7)$ \\
\hline Shallow (4) & $8(26)$ & $8(12)$ \\
\hline Shallow (20) & $8(26)$ & $8(12)$ \\
\hline Shallow (100) & $8(26)$ & $8(12)$ \\
\hline Shallow (500) & $8(26)$ & $8(12)$ \\
\hline Shallow (1000) & $8(26)$ & $8(12)$ \\
\hline Total & $2699(7246)$ & $1431(3326)$ \\
\hline & & \\
\hline & & \\
\hline
\end{tabular}




\section{References}

1) AL-Bayati; A.4. and lahif, I. S. (Anew 3-term PCG algorithm for non linear optimization problems) The American J. of Math. and Statist., USA, vol (4), no(2) pp81-87, (2008).

2) R. Fletcher, Practical Methods of optimization, second Edition, Wiley, chichester, England,1987.

3) M. R. Hestenes, and Stiefel E. "Methods of Conjugate Gradients for Solving Linear Systems" Journal of Research of the National Burean of Standards, Vol. 49, pp:409-436,1952

4) G. H. GoluBandc. and C. F. Vanloan. "Matrix computation", Johns Hopkins University Press, Baltimore, Maryland, 3nd edition,1996.

5) R. Fletcher, Practical Methods of optimization. Chichester Wiley, second Edition., 1987.

6) P. E. Frandsen, K. Jonasson, H. Nielsen and O. Tingleff, "Informatics and Mathematical Modelling", Technical University of Denmark, 3rd Edition, March 2004.

7) M. C. Biggs, "A note of minimization Algorithms which make use of non-Quadratic properties of the objective Function" journal of Institute of Mathematics and its Application vol.12, pp.337-338, 1973.

8) S. S. Oren, "self -scaling variable metric algorithms", Part II, Management Science, vol. 20, pp. 863-874, 1974.

9) S. S. Oren and D. G. Luenberger, "self-scaling variable metric (SSVM) Algorithms", part I, criteria and Sufficient conditions for scaling a class of Algorithms, Management science vol. 20, pp. 845865, 1974.

10) L. Armijo, "Minimization of functions having Lipchitz continuous first partial derivative", Pacific Journal of Mathematics, vol. 6, pp.13, 1966. 